\title{
Ultrasonic tomography of green wood using a non-parametric imaging algorithm with reflected waves
}

\author{
Loïc Brancheriau ${ }^{1 *}$, Philippe LAsaygues ${ }^{2}$, Eric DeBIEU $^{2}$, Jean Pierre LefebVrE ${ }^{2}$ \\ ${ }^{1}$ Agricultural Research Centre for International Development, UPR CIRAD 40, Production and Processing of Tropical Woods, \\ 34398 Montpellier Cedex 5, France \\ ${ }^{2}$ Laboratory of Mechanics and Acoustics, UPR CNRS 7051, 13402 Marseille Cedex 20, France
}

Keywords:

tomography /

ultrasound /

wood imaging

(Received 13 March 2008; accepted 6 June 2008)

\begin{abstract}
- Ultrasonic computed tomography in reflection was used to assess the integrity of green wood. Qualitative reflectivity images were obtained from back-scattered measurements by reflection tomography, like ultrasound in medical applications.

- The reconstruction algorithm was designed using, in particular, a linear approximation of the forward problem (Born approximation) and based on the assumption that a transversal cross section of wood is isotropic. The experimental device was composed of only one rotating emitter-receiver transducer to record and compute the projections. In this specific case, a tomographic projection was directly associated with a recorded signal. The qualitative aspect of this imaging technique was validated by performing a numerical simulation and tested on a small diameter green wood (Picea abies) $\log$.

- The images obtained were geometrically accurate considering the internal inclusions. It was, however, not possible in the simulation to differentiate the object shape from the background (coupling medium) because the reflectivity value associated with the object was too low. The image obtained with the spruce sample mainly showed the position of the bark as indicated by a very high contrast area. The proportion of transmitted energy was, however, sufficient to reconstruct the artificial inclusion within the sample.
\end{abstract}

\footnotetext{
Résumé - Tomographie ultrasonore de bois vert par reconstruction d'image non paramétrique en réflexion.

- L'imagerie tomographique ultrasonore en réflexion a été employée pour évaluer l'intégrité de bois à l'état vert. La tomographie en réflexion a permis d'obtenir des images qualitatives de réflectivité à partir de mesures rétro - diffusées analogues à l'échographie en médecine.

- L'algorithme de reconstruction était conçu en utilisant notamment une approximation linéaire du problème direct (l'approximation de Born) et en supposant que la section transverse du bois était isotrope. Le dispositif expérimental était composé d'un seul transducteur émetteur - récepteur en rotation pour enregistrer et calculer les projections. Dans ce cas spécifique, une projection tomographique était directement associée au signal enregistré. L'aspect qualitatif de cette technique d'imagerie a été validé en effectuant une simulation numérique et a été testé sur un rondin de faible diamètre à l'état vert (Picea abies).

- Les images obtenues étaient géométriquement justes en se référant aux inclusions internes. Il n'a cependant pas été possible lors de la simulation de discriminer l'objet de l'arrière plan (milieu couplant) en raison de la trop faible valeur de réflectivité associée à l'objet. L'image obtenue avec l'échantillon d'épicéa a principalement montré la position de l'écorce marquée par un très fort contraste. La proportion d'énergie transmise a cependant été suffisante pour reconstruire l'inclusion artificielle à l'intérieur de l'échantillon.
}

\footnotetext{
* Corresponding author: loic.brancheriau@ cirad.fr
} 


\section{INTRODUCTION}

Acoustic properties such as ultrasonic attenuation or velocity have been reported to reflect the strength of wood (Bucur, 1995) and predict in situ decay processes (Dunlop, 1981). However, ultrasound methods currently available for assessing the green wood status are limited to obtaining measurements in transmission mode through a section. These methods therefore give average values recorded along the ultrasound pathway through the section, but they are not suitable for assessing spatially heterogeneous acoustic properties of wood. Ultrasonic computed tomography (UCT) could thus be used in this setting to achieve more accurate wood quality assessment. UCT has a very large field of applications in decay diagnosis (Martinis, 2002) in standing trees in parks and public gardens, in lumber structure imaging (Neuenschwander et al., 1997) to reveal knots, grain deviations, cracks and compression wood, and in imaging defects in wood composites, such as delaminations between layers and voids (Bucur, 2003). Other wood imaging techniques exist, ranging from ionizing radiation, thermal techniques, microwave, ultrasound to nuclear magnetic resonance. The most relevant wood imaging technique depends upon the specific application (Bucur, 2003).

Although UCT is a valuable tool for imaging soft biological materials (Mensah et al., 1997), little attention has been paid to using this method for imaging harder media such as green wood. Dikrallah published preliminary results of an analysis of the anisotropy effect on acoustic wave propagation in green wood (Dikrallah et al., 2006). In an important study, Maurer also highlighted the anisotropy effect and proposed a correction procedure for quantitative tomography (Maurer et al., 2006). Interactions between ultrasound and wood, especially green wood, make the imaging process particularly intricate. For instance, classical reconstruction algorithms are based on the assumption that straight ray propagation is involved, whereas the incident beam is liable to be refracted at the interface between the soft background (coupling gel, water) and the wood, depending on the incident angle, due to the marked difference between the acoustic properties of the two media. Diffraction - which is closely related to refraction - can also result from the irregular shape of the samples. Moreover, specific reconstruction tools are sometimes required due to the anisotropy of ultrasound propagation through green wood (Maurer et al., 2005; 2006).

Many difficulties may be encountered when attempting to assess wood by quantitative ultrasonic tomography. However, UCT imaging is an attractive non-invasive tool that can be used to perform quantitative 3-D reconstruction of the tree geometry and determine acoustic properties such as ultrasound velocity and attenuation, depending on the type of measurements (time-of-flight or amplitude) performed. In previous studies (Martinis et al., 2004; Nicolotti et al., 2003), projection data were obtained in transmission mode through a wood specimen, which could be inversed by using classical tomographic reconstruction algorithms (Kak et al., 1988). Previously published preliminary results showed that tomographic reconstruction of an academic test object and green wood was feasible using ultrasonic transmission tomography techniques
(Martinis et al., 2004; Maurer et al., 2005; Socco et al., 2004). The authors therefore hypothesized that reconstruction of the outer boundary of samples and the inner boundaries of different decay regions would provide information on the wood strength.

However, these studies were seriously hampered by several factors. First, the boundaries of the object could not be easily reconstructed from datasets acquired in transmission mode, but they could from datasets acquired in reflection mode, which gives an improved tomographic view of the scatter shape. Furthermore, the mesh in transmission-UCT is often too large, and cavities of less than $50 \mathrm{~mm}$ diameter are generally imperfectly identified (Martinis et al., 2004; Nicolotti et al. 2003). Secondly, when inversing the dataset acquired in transmission mode to recover the sound velocity image of a cross-section, the signals have to be clearly identified, and the time-of-flight must be measured accurately from a signal with a low signal-to-noise ratio. Complex interactions between the incident ultrasonic wave field and wood give rise to a complex transmission field with several output signals, which can make it difficult to identify the signals.

The main objectives of our study were first to take full advantage of UCT in diffraction and transmission modes by developing a technique for cross-sectional imaging of wood, and secondly to refine the signal processing technique by developing a suitable signal filtering process based on wavelet decomposition to improve assessments based on time-of-flight measurements.

The present paper focuses on the qualitative (nonparametric) image obtained using reflection-UCT to detect decay in green wood. Reflection-UCT, which is derived from the general diffraction-UCT approach, involves forming an image which highlights the interfaces between media of different acoustic impedance from backscattered measurements. One interesting aspect of reflection tomography in comparison with transmission tomography is that it is not necessary to encompass the object with transmitters and receivers for collection of projection data collection. Transmission and reception of signals are performed by the same transducer. However, since most of the ultrasonic energy is scattered in the forward direction, the transducer must have high sensitivity in order to measure backscattered signals at high signal-to-noise ratios.

We perform a numerical simulation to study the effects of several decay patterns and "artificial" wood configurations on the reflection-UCT data obtained. The experimental test performed on a green wood sample using a tomographic scanner revealed the advantages and limitations of the classical algorithms.

\section{MATERIALS AND METHODS}

\subsection{Experimental set-up}

The experimental setup was designed to perform all types of ultrasonic measurements (in reflection, diffraction and transmission). Only one emitter-receiver transducer was used in reflection tomography. It included an acoustic device that could be moved with 


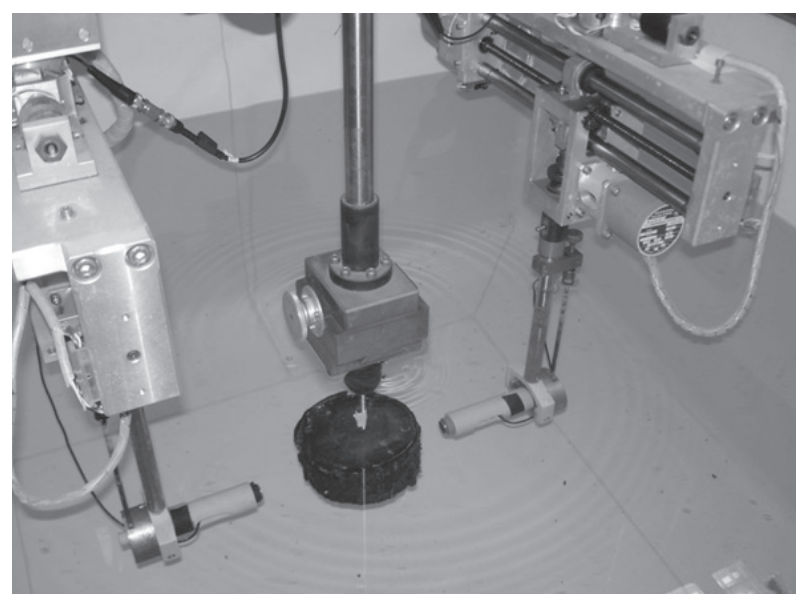

Figure 1. Details of the experimental device for measurements in reflection, diffraction and transmission. Only one emitter-receiver transducer was used in reflection tomography.

various degrees of freedom and used to analyze samples in all directions (Fig. 1). Ultrasounds were generated using a Panametric ${ }^{\circledR}$ pulse/receiver and Imasonic ${ }^{\circledR}$ piezo-composite wide-band transducers with a nominal frequency of $0.5 \mathrm{MHz}$. The object under investigation was placed in the center of the measuring system. The complete circle area was scanned using a $5^{\circ}$ sampling angular increment, so 72 projections were obtained. Each projection, equal to each backscattered signal in reflection tomography, was composed of 4096 samples regularly spaced at intervals of $50 \mathrm{~ns}(20 \mathrm{MHz})$. The upper limit of the number of pixels $N$ (1) that is possible to use for reconstruction was thus $384 \times 384$ pixels (Socco et al., 2004).

$$
N=\frac{N_{\text {Projection }} \times N_{\text {Sample }}}{2}
$$

with $N_{\text {Projection }}$ : the number of projections; $N_{\text {Sample }}$ : the number of samples per projection. The chosen size of the tomographic image was $300 \times 300$ pixels, which corresponds to the inner square of the scanned area with an associated spatial resolution of $1 \mathrm{~mm}^{2}$ scanned per pixel (the rotation radius of the transducer is equal to $22 \mathrm{~cm}$ with the object situated in the far field of the ultrasonic emission). The longitudinal resolution (along the emission axis) is the wavelength in the object and was $3 \mathrm{~mm}$. The lateral resolution (perpendicular to the emission axis) was $18 \mathrm{~mm}$ using the Fresnel zone formula (2) (Socco et al., 2004).

$$
R_{F}=\sqrt{\frac{S_{d} c}{2 f}}
$$

with $R_{F}$ : the Fresnel zone radius; $S_{d}$ : the scanned depth (the rotation radius of the emitter-receiver transducer); $c$ : the celerity; $f$ : the nominal frequency of the transducer.

\subsection{Ultrasonic computed tomography}

The aim of UCT is to reconstruct the spatial distribution of some geometrical and physical parameters of an object from scattered ultrasonic measurements. These measurements are carried out on sets of transmitter and receiver positions spaced at different densities, with various interrogating wave frequencies. We are then faced both with a forward scattering problem, i.e. predicting the pressure field when the scattering medium and incident field are assumed to be known, and with the inverse scattering problem, i.e. retrieving parameters of the medium from the measured incident and scattered fields. This inverse problem is non-linear and ill posed and there is generally no single solution. It is essential to find a way of eliminating solutions that do not reflect the real situation.

\subsubsection{Forward problem modeling}

Let $A$ be the operator that describes elastic wave propagation or scattering phenomena in the heterogeneous medium that is to be imaged (3), including boundary and Sommerfeld conditions (Morse and Ingard, 1968).

$$
\text { A. }=-\frac{1}{c^{2}} \frac{\partial^{2}}{\partial t^{2}}+\rho \operatorname{div}\left(\frac{1}{\rho} \text { grad. }\right)
$$

with $\rho$ : the variable density, and $c$ : the variable celerity.

Let $S$ be the ultrasonic source (transducer emission), which is assumed to be known. The resulting ultrasonic field $\varphi$ satisfies Equation (4):

$$
A \varphi=S
$$

The heterogeneous medium is assumed to be composed of a known part, i.e. the coupling medium associated with the wave operator $A_{0}(5)$, and an unknown part, i.e. the object associated with the operator $A^{\prime}$. The relation between $A, A_{0}$ and $A^{\prime}$ is given by Equation (6).

$$
A_{0}=-\frac{1}{c_{0}^{2}} \frac{\partial^{2}}{\partial t^{2}}+\Delta .
$$

with $c_{0}$ : the constant celerity in the coupling medium. $\Delta .:$ the Laplacian operator.

$$
A=A_{0}+A^{\prime}
$$

Solution $\varphi_{0}$ of the non-perturbed problem is assumed to be known:

$$
A_{0} \varphi_{0}=S
$$

Field $\varphi^{\prime}$ induced by perturbation of the object can be thus written as the difference between $\varphi$ and $\varphi_{0}\left(\varphi^{\prime}=\varphi-\varphi_{0}\right)$. Then $\varphi^{\prime}$ is the solution of Equation (8):

$$
A_{0} \varphi^{\prime}=-A^{\prime} \varphi
$$

If Green's function $G_{0}$ of Equation (5) is known $\left(G_{0}\right.$ solution of $A_{0} G_{0}=\delta$ ), the field induced by the object is given by Equation (9).

$$
\varphi^{\prime}=-G_{0} * A^{\prime} \varphi
$$

In this equation, the operator " $*$ " is the convolution product. Equation (9) is the well-known Lippmann-Schwinger equation (Morse and Ingard, 1968).

\subsubsection{Linearization and inverse problem in reflection}

The inverse problem is the solution of the nonlinear Equation (9) and a solution can be found by means of a perturbation scheme (based on a linear approximation). The first-order Born approximation is one of these schemes (Born series) and the first-order solution $\varphi^{\prime(1)}$ can be written:

$$
\varphi^{\prime(1)}=-G_{0} * A^{\prime} \varphi_{0}
$$


The far-field solution of Equation (10) in direction $\vec{n}$ for plane wave insonification in direction $\overrightarrow{n_{0}}$ is:

$$
\varphi^{\prime(1)}(x, \omega)=-\frac{e^{i k|\vec{x}|}}{4 \pi|\vec{x}|} h^{(1)}\left(\overrightarrow{n_{0}}, \vec{n}, \omega\right)
$$

With $h^{(1)}\left(\overrightarrow{n_{0}}, \vec{n}, \omega\right)=k^{2}\left[\left(1+\overrightarrow{n_{0}} \cdot \vec{n}\right) \hat{\alpha}(\vec{K})+\left(1-\overrightarrow{n_{0}} \cdot \vec{n}\right) \hat{\xi}(\vec{K})\right]$ and $\vec{K}=$ $k\left(\vec{n}-\overrightarrow{n_{0}}\right) \cdot k=\omega / c_{0}$ : representing the wave number in the reference medium. $\hat{\alpha}(\vec{K})$ and $\hat{\xi}(\vec{K})$ : the spatial Fourier transforms of $\alpha=\frac{c^{2}-c_{0}^{2}}{c^{2}}$, the quadratic fluctuation of celerity, and $\xi=\ln \left(\frac{Z}{Z_{0}}\right)$, the logarithmic fluctuation of acoustic impedance $z=\rho c \cdot h^{(1)}\left(\overrightarrow{n_{0}}, \vec{n}, \omega\right)$ : the first order frequency response (or transfer function) of the medium for incidence $\overrightarrow{n_{0}}$ and direction of observation $\vec{n}$.

In the back-scattering configuration used in reflection mode $\vec{n}=$ $-\overrightarrow{n_{0}}$ so that the transfer function becomes:

$$
h^{R(1)}\left(\overrightarrow{n_{0}}, \omega\right)=2 k^{2} \hat{\xi}(\vec{K}) \quad \text { with } \quad \vec{K}=-2 k \overrightarrow{n_{0}}
$$

Equation (12) shows that the only parameter that we can hope to attain by back-scattering measurements is the logarithmic fluctuation of the acoustic impedance. Parameter $\xi$ is directly linked to the reflectivity of the object using the following first-order development (13). The acoustic impedance contrast is defined as $\left(z-z_{0}\right) / z_{0}$.

$$
\xi(x)=\ln \left(\frac{z(x)}{z_{0}}\right) \approx \frac{z(x)-z_{0}}{z_{0}} \approx 2 r(x)
$$

with $r(x)=\frac{z(x)-z_{0}}{z(x)+z_{0}}$ : being the spatial reflectivity coefficient of the object.

The aim of ultrasonic reflection tomography is thus to obtain qualitative reflectivity images from back-scattered measurements. The situation is identical to that of X-ray tomography (the source rotates around the object and the projections are recorded after each rotation step), but with a band-pass spectral filter instead of a low-pass spectral filter (because piezoelectric ultrasonic transducers cannot transmit low frequencies). The reconstruction is performed using the summation of ramp-filtered back-projections (Franceschini et al., 2007; Mensah et al., 2007):

$$
|\Delta r(x, y)|=\frac{1}{4 \pi} \int_{0}^{2 \pi} \pi_{\phi}(l) d \phi
$$

with $(x, y)$ : the spatial coordinates along the horizontal and perpendicular axis; $l$ : the coordinate along the emission axis $\overrightarrow{n_{0}} ; \phi:$ the angle formed by the emitter - receiver transducer (emission axis) and the horizontal axis. The vector $\pi_{\phi}(l)$ results from filtering of the projection $P_{\phi}(l)$ by a ramp filter. Each projection $P_{\phi}(l)$ is formed by each received signal; a reflected signal is indeed formed by amplitudes directly linked with the local reflectivity coefficients (along with the local attenuations). Equation (14) shows that the inversion procedure computes the modulus of the Laplacian of the reflectivity; only outlines of the object areas (interfaces between media of different acoustic impedance) that induce variations in impedance contrast are thus reconstructed. This last remark explains why this specific reflection tomographic technique produced qualitative and not quantitative images.

\subsubsection{Assumptions adopted in UCT for green wood imaging}

Weak scattering approximations are generally used when imaging soft low-contrast media such as biological tissues, e.g. the approximations of Born or Rytov (Greenleaf, 1970). Contrary to what occurs with soft media, the acoustic contrast between wood and the

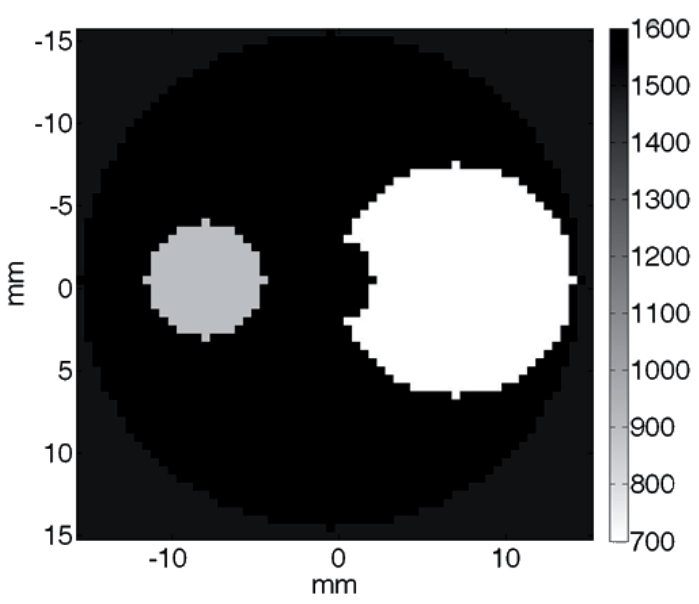

Figure 2. Numerically simulated test target. The bar indicates the wave velocity value (in $\mathrm{m} / \mathrm{s}$ ).

surrounding reference medium (coupling gel or water) is so great that the problem is intrinsically non-linear in terms of the unknown physical and geometrical parameters, and there is generally no single solution. One solution therefore involves finding the homogenized or "equivalent" material parameters of a spatially bounded heterogeneous medium, and adapting the acquisition and reconstruction software initially developed for use with soft media.

Trees consist of long circular cylinders surrounded by an irregular thick bark. These cylinders are generally assimilated to orthotropic materials with a principal axis of symmetry oriented along the vertical axis (Goodman et al., 1970). In this study, transversal cross-sections of the wood to be imaged were taken to be isotropic and therefore assumed to mediate the propagation of more complex waves, similar to volume waves. Since the typical compression bulk wave velocity recorded in the transverse direction was $1400-1600 \mathrm{~m} / \mathrm{s}$, the wavelength at the frequency used here $(\sim 0.5 \mathrm{MHz})$ was around $2.8-$ $3.2 \mathrm{~mm}$, which is much larger than the microscopic structural heterogeneities $(\sim 10 \mu \mathrm{m})$. Reflections induced by annual growth rings cannot be neglected $(\sim 1 \mathrm{~mm})$ and can form a specific geometric pattern in the tomographic image. The first-order Born approximation solution could therefore bias the tomographic reconstruction.

The wavelength in water $(\sim 3 \mathrm{~mm})$ was much shorter than the diameter of the object (approximately 100-200 mm). Ray theory was applied and straight lines inside the object were taken to approximate the wave propagation paths. Since signal contributions associated with compression waves were only of interest here, a weak nonhomogeneous fluid could be used to model wood.

\subsection{Methodology}

Reflection-UCT was applied to a numerically simulated test target and to a green wood sample. The numerically simulated test target was defined as a homogeneous and isotropic circular $30 \mathrm{~mm}$ diameter cylinder (Fig. 2, $50 \times 50$ pixels). The mean velocity of the compression wave was set at $c_{0}=1500 \mathrm{~m} / \mathrm{s}$ in water (associated acoustic impedance $z_{0}=1.5 \times 10^{6}$ N.s.m ${ }^{-3}$ ) and $c=1600 \mathrm{~m} / \mathrm{s}$ in the material $\left(z=1.60 \times 10^{6} \mathrm{~N} . \mathrm{s}^{-3} \mathrm{~m}^{-3}\right)$. This latter velocity corresponds to the same scale as in a transversal cross section of a tree (in dry condition). The sample contained two $7 \mathrm{~mm}$ and $14 \mathrm{~mm}$ diameter 


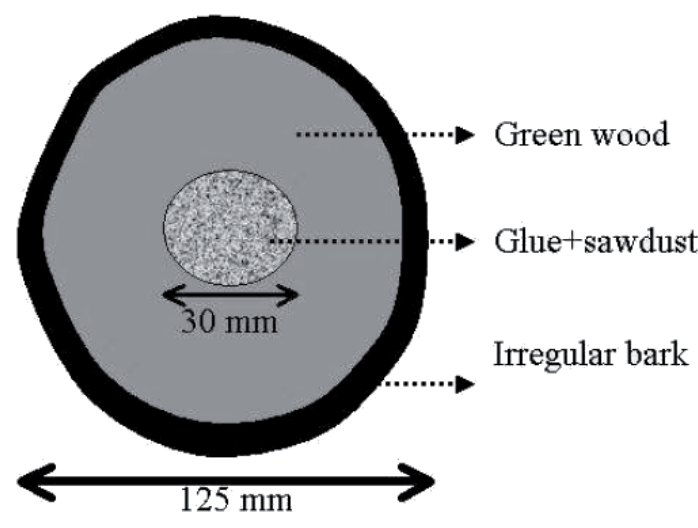

Figure 3. Spruce (Picea abies) sample geometry.

inclusions, corresponding to the presence of decay $\left(c_{1}=700 \mathrm{~m} / \mathrm{s}\right.$, $z_{1}=0.70 \times 10^{6}$ N.s.m ${ }^{-3}$ and $c_{2}=1300 \mathrm{~m} / \mathrm{s}, z_{2}=1.30 \times 10^{6}$ N.s.m $\left.{ }^{-3}\right)$.

The spruce sample (Picea abies) was green, surrounded by bark, and measured $125 \mathrm{~mm}$ diameter and $200 \mathrm{~mm}$ thick. A mixture of glue and sawdust was placed in a $30 \mathrm{~mm}$ hole drilled in the center of the sample to create artificial decay (Fig. 3). The mean moisture content, measured on a disk from the same specimen, was $110 \%$. The velocity decreases with increasing moisture content. The maximum velocity corresponds to the dry condition and the decreasing trend is linear up to the fiber saturation point (due to the combined effects of the decreased modulus of elasticity and the increased density). After this point, there is a trend inflection and the velocity continues to decline linearly due only to the increased density (Bucur, 1995). Considering the influence of moisture content on the sample acoustic impedance, the $z=0.55 \times 10^{6}$ N.s.m ${ }^{-3}$ value measured in dry condition $(12 \%$ moisture content, $1400 \mathrm{~m} / \mathrm{s}$ mean velocity and $390 \mathrm{~kg} / \mathrm{m}^{3}$ density) is quasi-equal to the $z=0.59 \times 10^{6} \mathrm{~N} . \mathrm{s} . \mathrm{m}^{-3}$ value measured in wet condition $\left(110 \%\right.$ moisture content, $860 \mathrm{~m} / \mathrm{s}$ mean velocity and $690 \mathrm{~kg} / \mathrm{m}^{3}$ density). The velocity and density measurements were obtained on a prismatic sample from the same specimen. The $z=0.59 \times 10^{6}$ N.s.m ${ }^{-3}$ value was then used as the a priori value for the specimen.

\section{RESULTS AND DISCUSSION}

\subsection{Numerical simulation}

In order to perform the reconstruction, 180 projections of 70 samples were simulated $\left(2^{\circ}\right.$ angular increment). The upper limit of the reconstructed image size was thus $79 \times 79$ pixels, but the final tomographic image size was equal to the original size of the test target $(50 \times 50$ pixels). Each projection was set to be sampled at $1 \mathrm{MHz}$ (1 pixel per $\mu$ s in Fig. 4). The nominal frequency of ultrasonic waves was $0.5 \mathrm{MHz}$ (the $1 \mathrm{MHz}$ sampling frequency was equal to the limit of the Nyquist - Shannon sampling theorem). The simulated duration of acquisition was $70 \mu \mathrm{s}$, which corresponded to the maximum propagation time in reflection.

Figure 4 gives the ultrasonic sinogram formed by 180 simulated signals in reflection (ray theory was applied without taking attenuation in the different media into account; to simplify Figure 4, only the signal envelopes are shown). Figure 5 presents the associated reconstructed image of the numerically

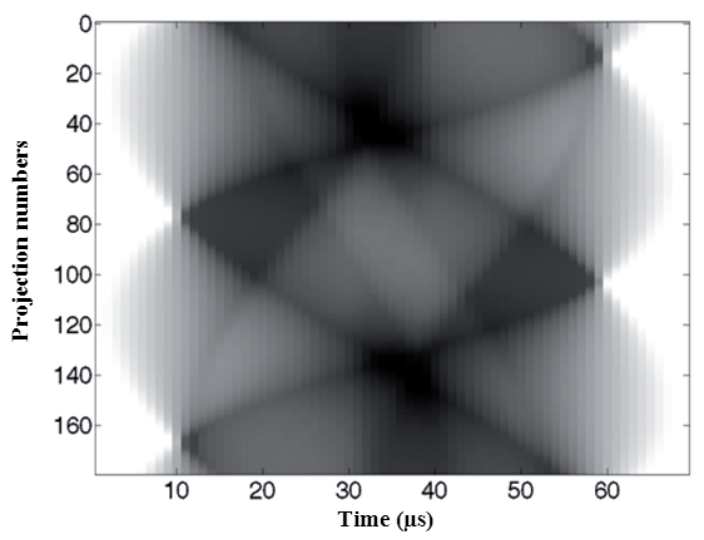

Figure 4. Simulated ultrasonic sinogram. The color corresponds to the signal envelope magnitude. A recorded signal corresponds to a projection.

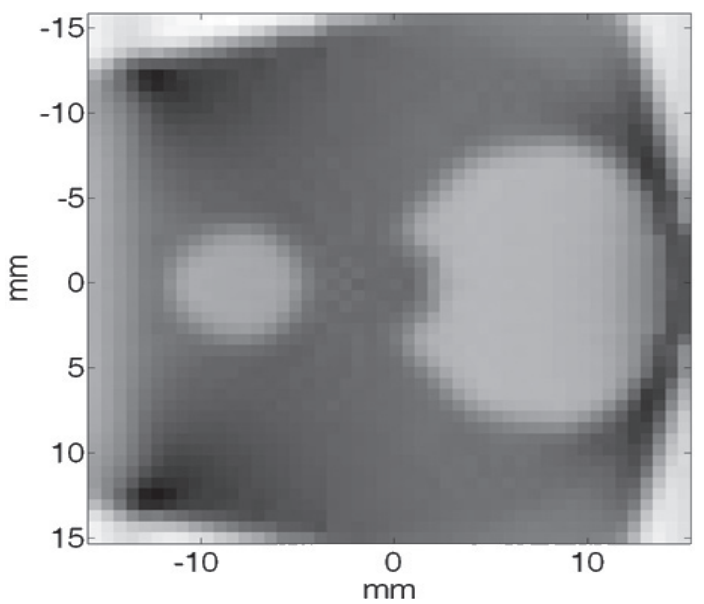

Figure 5. Simulated ultrasonic tomogram. The color difference corresponds to the contrast between areas of different acoustic impedance (modulus of the Laplacian of the reflectivity).

simulated test target. In this figure, the transition areas highlighting the interface positions were 6 pixels thick $(3.6 \mathrm{~mm}$ associated spatial resolution). This resolution was in accordance with the 5 pixel theoretical resolution value $(3 \mathrm{~mm}$ wavelength). The diameters of the reconstructed inclusions were 12 pixels (12 pixel original diameter of inclusion 1) and 27 pixels (24 pixel original diameter of inclusion 2), while taking the center of the transition area as the interface position. Figure 5 was thus geometrically accurate with respect to the actual inclusion dimensions.

The result was acceptable since the Born approximation was used (resolution close to the theoretical value), and the background consisted of water with an a priori wave velocity of $1500 \mathrm{~m} / \mathrm{s}$. The perturbation in this case was introduced by the two holes simulating decay. The magnitude of the reflectivity coefficient associated with the two inclusions was $\left|r_{1}\right|=$ 0.36 (7 mm diameter inclusion) and $\left|r_{2}\right|=0.07$ (14 mm diameter inclusion). The most highly contrasted area was therefore 


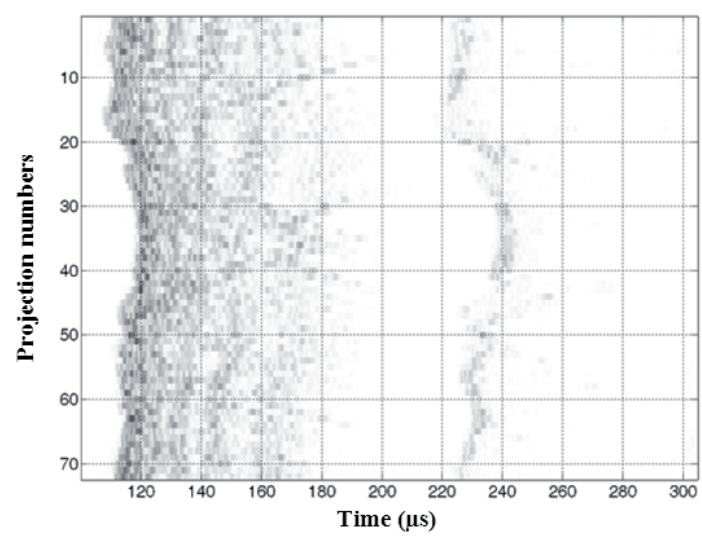

Figure 6. Reflection-UCT of a spruce (Picea abies) sample with centered artificial decay, ultrasonic reflection sinogram.

naturally focused on the inner holes rather than on the external boundaries of the target. In this example, the bark was not simulated, and there was very little difference in velocity between the water and the material $(100 \mathrm{~m} / \mathrm{s})$. The reflectivity coefficient value associated with the water-material interface was $r=0.03$, which meant that only $0.1 \%$ of the wave energy was reflected by this interface. It was therefore not possible for the algorithm to discriminate between the two domains. The reference background used for the inversion procedure was finally the set composed of the water and the main material. Since the difference in impedance contrast between the material and the holes increased, they could be easily identified in this example.

\subsection{Wood sample}

Figure 6 shows the ultrasonic sinogram formed by 72 signals in reflection. The difficulties encountered were mainly due to the high wave attenuation during propagation (Fig. 6). Signals were therefore amplified but not saturated. Figure 7 shows the reconstructed reflection-UCT of a spruce sample (Picea abies). Besides the wave attenuation during propagation, note that the bark had very marked effects on the signals. A large amount of air was probably sandwiched between the healthy tree and the bark. The acoustic impedances were very different, which affected the wave propagation by a sudden interruption. Given the surface texture irregularity, it is likely that only a small part of the incident energy penetrated into the wood, and a large proportion was directly backscattered without penetrating into the healthy wood. This assumption is in line with the strong echoes, which could be taken to reflect the bark thickness when not attenuated between the healthy wood and the bark.

A number of 36 tomographic profiles were extracted from the reconstructed image (Fig. 7) by rotation around the center of the image with a $5^{\circ}$ angular increment. Figure 8 shows an example of a tomographic profile obtained. The wooden sample diameter was correctly assessed (125 mm, Fig. 8), and the inclusion was well positioned in the center, but it was impossible to measure its diameter very precisely with a resolution

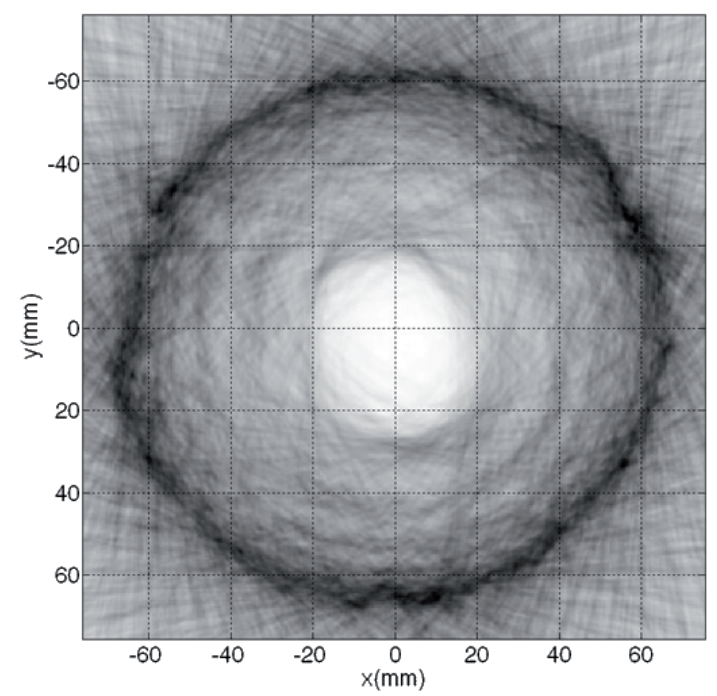

Figure 7. Reflection-UCT of a spruce (Picea abies) sample with centered artificial decay, ultrasonic reflection tomogram.

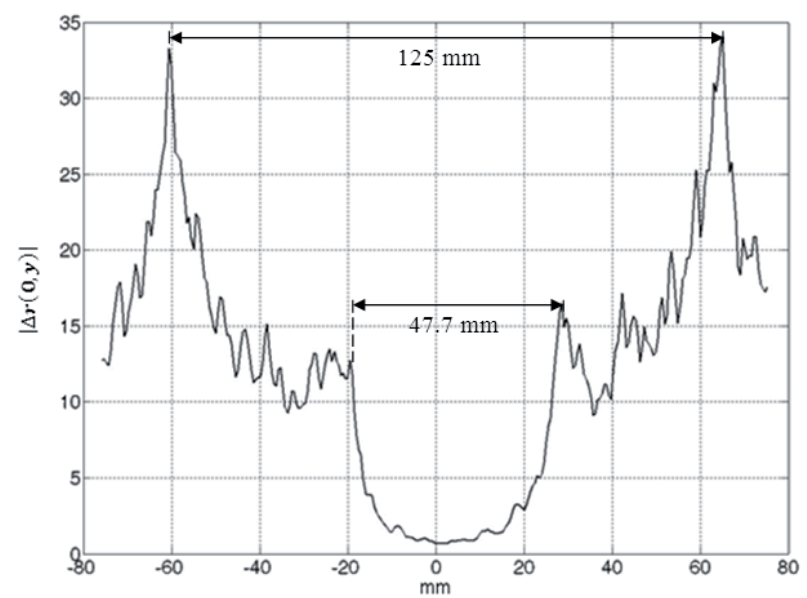

Figure 8. Reflection-UCT of a spruce (Picea abies) sample with centered artificial decay, y axis profile of the ultrasonic reflection tomogram (modulus of the Laplacian of the reflectivity at $x=0 \mathrm{~mm}$ ).

close to the wavelength (Fig. 8). The measurement of the inclusion diameter was carried out by an operator on the 36 profiles. The average diameter was $48.1 \mathrm{~mm}$ (minimum value: $39.1 \mathrm{~mm}$, maximum value: $57.8 \mathrm{~mm}$ and standard deviation: $4.0 \mathrm{~mm}$ ) instead $30 \mathrm{~mm}$ (the true value). The difference between the measured value and true value allowed us to estimate the spatial resolution obtained; the resolution value was around $20 \mathrm{~mm}$, which was very close to the theoretical Fresnel zone radius of $18 \mathrm{~mm}$ (lateral resolution). The image obtained in Figure 7 and the profile in Figure 8 were blurred by several cumulated effects: the Born approximation was used despite the strong echo produced by the bark, the cross section was assumed to be isotropic and the lateral resolution was 6-fold higher than the longitudinal resolution. 


\section{CONCLUSION}

A wood qualitative imaging method based on ultrasonic computed tomography analysis of soft tissues was presented. Due to the acoustic properties of wood (attenuation and impedance contrast), working with this material is more complex than dealing with soft tissues. Wood was assumed to be equivalent to an internally weakly contrasted object (local fluctuations in the acoustic characteristics of a cross-section are weak) immersed in a homogeneous reference medium (water). The first-order Born approximation was used to solve the inverse problem of image reconstruction. The main result of the theoretical inversion was that only the interface outlines in the object were reconstructed; this specific reflection tomographic technique produced qualitative but not quantitative images. The transversal cross-sections of the wood to be imaged were taken to be isotropic. Consequently, ray theory was applied and straight lines within the object were considered to approximate the wave propagation paths.

The reconstruction algorithm was tested on an academic object produced by numerical simulation. The image obtained was geometrically accurate considering the two simulated inclusions. The computed inclusion positions and diameters were equal to those of the original object, with a spatial resolution that was in accordance with the theoretical resolution. It was, however, not possible to differentiate the object shape from the background (coupling medium). This phenomenon could be explained by the fact that the reflectivity value of the interface associated with the transition object-background was too low. A green spruce sample with bark was also tested. Artificial decay made with glue and sawdust was created at the center of the sample. The image obtained mainly showed the position of the bark indicated by very high contrast. It seemed that a large proportion of the incident energy was directly backscattered by the bark without going deeper into the material. Despite this phenomenon, the proportion of transmitted energy was sufficient to reconstruct the artificial inclusion within the sample. This inclusion was well positioned at the center, but an $18 \mathrm{~mm}$ spatial resolution evaluation made the diameter estimation very inaccurate.

This research could now be continued in two possible areas. The first approach could be based on the Born iterative method, where iterations are calculated numerically from experimental data (Lasaygues et al., 2006). The second possible approach could involve modeling the forward problem using the intercepting canonical body approximation method, which reduces the calculation cost. The latter method involves taking the wood covered with bark and the decay it contains to be a non-circular multilayer cylinder in which every layer (bark, growth rings, decay) is homogeneous. Contrary to approaches based on straight ray theory, this method requires no limited approximations and no a priori information. The scattered field is completely integrated (Franceschini et al., 2007).

\section{REFERENCES}

Bucur V., 1995. Acoustics of wood, CRC Press Inc., Boca Raton, 284 p.

Bucur V., 2003. Nondestructive Characterization and Imaging of Wood, Springer-Verlag, Berlin, $324 \mathrm{p}$.

Dikrallah A., Hakam A., Kabouchi B., Brancheriau L., Baillères H., Famiri A., and Ziani M., 2006. Experimental analysis of acoustic anisotropy of green wood by using guided waves. Proceedings of the ESWM-COST Action E35, Florence, Italy, pp. 149-154.

Dunlop J.I., 1981. Testing of poles by using acoustic pulse method. Wood Sci. Technol. 15: 301-310.

Franceschini E., Mensah S., Le Marrec L., and Lasaygues P., 2007. An optimization method for quantitative impedance tomography. IEEE Trans. Ultrason. Ferroelec. Freq. Control. 54: 1578-1588.

Goodman J.R. and Bodig J., 1970. Orthotropic Elastic Properties of Wood. J. Struct. Div. 96: 2301-2319.

Greenleaf F., 1970. Introduction to Computer Ultrasound Tomography. Proceedings of the IFIP TC-4 Working Conference, Computed Aided Tomography and Ultrasonics in Medicine, North-Holland, pp. $125-136$.

Kak A.C. and Slaney M., 1988. Principles of computerized tomographic imaging. IEEE press, $329 \mathrm{p}$.

Lasaygues P., Guillermin R., and Lefebvre J.-P., 2006. Distorted Born Diffraction Tomography: limits and applications to inverse the ultrasonic field scattered by an non-circular infinite elastic tube. Ultrason. Imaging 28: 211-229.

Martinis R., 2002. Nondestructive techniques for decay diagnosis on standing trees. Thesis University of Florence, Italy, $300 \mathrm{p}$.

Martinis R., Socco L.V., Sambuelli L., Nicolotti G., Schmitt O., and Bucur V., 2004. Tomographie ultrasonore pour les arbres sur pied. Ann. For. Sci. 61: 157-162.

Maurer H.R., Schubert S.I., Baechle F., Clauss S., Gsell D., Dual J., and Niemz P., 2005. Application of nonlinear acoustic tomography for non-destructive testing of trees. Proceedings of the 14th international symposium on non-destructive testing of wood, Hannover, Germany, pp. 339-349.

Maurer H.R., Schubert S., I., Baechle F., Clauss S., Gsell D., Dual J., and Niemz P., 2006. A simple anisotropy correction procedure for acoustic wood tomography. Holzforschung 60: 567-573.

Mensah S. and Lefebvre J.P., 1997. Enhanced Diffraction Tomography. IEEE Trans. Ultrason. Ferroelec. Freq. Control. 44: 1245-1252.

Mensah S. and Franceschinie E., 2007. Near-field ultrasound tomography. J. Acoust. Soc. Am. 121: 1423-1433.

Morse P.M. and Ingard K.U., 1968. Theoretical Acoustics, McGraw-Hill, New York, 913 p.

Neuenschwander J., Niemz P., and Kucera U., 1997. Studies for visualizing wood defects using ultrasonics in reflection and transmission modes. Holz Roh-Werkst. 55: 339-340.

Nicolotti G., Socco L.V., Martinis R., Godio A., and Sambuelli L., 2003. Application and comparison of three tomographic techniques for detection of decay in trees. J. Arboric. 29: 66-78.

Socco L.V., Sambuelli L., Martinis R., Comino E., and Nicolotti G., 2004. Feasibility of ultrasonic tomography for nondestructive testing of decay on living trees. Res. Nondestr. Eval. 15: 31-54. 